\title{
Estudio del brote epidémico de lagarta peluda (Lymantria dispar L.) en masas de Pinus radiata en Cubillos del Sil (El Bierzo, León)
}

\author{
Lago-Parra G. ${ }^{1}$, Castedo-Dorado F. ${ }^{1}$, Álvarez Taboada M.F. ${ }^{2}$ y Lombardero M.J. ${ }^{3}$ \\ ${ }^{1}$ Departamento de Ingeniería y Ciencias Agrarias, Escuela Superior y Técnica de Ingeniería Agraria, \\ Universidad de León, Campus de Ponferrada, Avda. de Astorga s/n, 24400, Ponferrada \\ (Gorka187@hotmail.com; fcasd@unileon.es)
}

${ }^{2}$ Departamento de Tecnología Minera, Topográfica y de Estructuras, Escuela Superior y Técnica de Ingenieros de Minas, Universidad de León, Campus de Ponferrada, Avda. de Astorga s/n, 24400, Ponferrada

(flor.alvarez@unileon.es)

${ }^{3}$ Departamento de Producción Vegetal, Escuela Politécnica Superior, Universidad de Santiago de Compostela, Campus de Lugo, Campus universitario s/n, 27002, Lugo

(mariajosefa.lombardero@usc.es)

\section{Resumen}

En este trabajo se presentan los resultados del seguimiento de un brote epidémico del insecto defoliador Lymantria dispar ocurrido en El Bierzo (León) en los años 2012 y 2013. La principal peculiaridad de este brote es que el insecto defolió fundamentalmente rodales de Pinus radiata, a pesar de existir en la zona otros huéspedes a priori más adecuados. Se estudió el papel de la planta huésped en el desarrollo del insecto mediante el análisis de la calidad nutritiva y palatabilidad de $P$. radiata frente a hojas de tres de sus huéspedes habituales (Quercus ilex, Castanea sativa y Populus nigra) presentes en la zona y mediante bioensayos de alimentación controlada con estas especies. Los resultados muestran que, ni la concentración de nitrógeno en las hojas, ni la dureza de las mismas, parecen explicar las ventajas nutritivas de $P$. radiata para $L$. dispar frente a las otras especies arbóreas estudiadas. A pesar de lo peculiar del hábitat estudiado, los enemigos naturales de $L$. dispar identificados son los habitualmente citados por otros autores en la Península Ibérica, con la excepción del depredador de huevos Dermestes erichsoni. Estos resultados sugieren que la especie huésped no ha interferido en la localización de $L$. dispar por parte de sus enemigos naturales. Mediante análisis de anillos de crecimiento se ha comprobado la existencia de una disminución significativa del crecimiento radial durante y tras la defoliación en árboles con nivel de defoliación severa (75-100\%) en comparación con árboles con nivel de defoliación muy baja o nula (0-25\%). Las claras sólo resultaron significativas en el crecimiento radial post-defoliación.

Palabras clave: defoliación, preferencias tróficas, enemigos naturales, crecimiento, Lymantria dispar, Pinus radiata. 


\section{Introducción}

Lymantria dispar L. (Lepidoptera, Lymantriidae) es un insecto defoliador cuyas larvas se alimentan de una gama muy amplia de huéspedes, entre los que destacan algunos géneros arbóreos como Quercus, Populus, Salix, Larix y Betula (Tobin y Liebhold, 2011). En España se puede encontrar además en Acer, Alnus, Arbutus, Castanea, Corylus, Fagus, Fraxinus y Pinus (Muñoz et al., 2011), mostrando una clara preferencia por Quercus ilex y $Q$. suber en regiones mediterráneas (Alalouni et al., 2013).

Según Twery (1990) y Liebhold et al. (1995), las especies forestales se pueden clasificar en tres grupos en función de su susceptibilidad a ataques de L. dispar: especies susceptibles, resistentes e inmunes a la defoliación. Las especies susceptibles son aquellas que son consumidas por todos los estados larvarios del insecto; las especies resistentes son aquellas que son consumidas sólo por algunos estados larvarios o cuando las especies susceptibles no están disponibles, y las especies inmunes son aquellas que muy rara vez son con consumidas por cualquier estado larvario. Según esta clasificación, se admite que, exceptuando las pináceas de hoja caduca (Larix spp., Metasequoia spp. y Taxodium spp.), la mayoría de las restantes pináceas se pueden catalogar como resistentes. Aun así, las referencias a defoliaciones en masas de coníferas de hoja perenne son relativamente comunes. Así, por ejemplo, Closa y Núñez (2009) documentan la existencia de daños en masas de $P$. pinaster y $P$. halepensis en Menorca provocadas por estados larvarios tardíos; Avci (2009) recoge la existencia de daños en Turquía sobre P. brutia y Cedrus libani en masas mixtas con frondosas, donde las quercíneas actuaron como huéspedes principales; Brown et al. (1988) y Eisenbies et al. (2007) indicaron la presencia de daños en $P$. taeda y P. strobus en masas mixtas con robles en el este de EE.UU.

En el caso concreto de masas de Pinus radiata, en España están documentados dos brotes epidémicos de L. dispar en los años 1952-1953, uno en Asturias y otro en Pontevedra (Romanyk y Rupérez, 1960; Romanyk, 1973). Por otra parte, en Portugal, Leites (1993) documentó un ataque que afectó a 290 ha de una masa de 15 años en el noroeste del país. Por último, Fraval (1989) evidenció un ataque a masas de Eucalyptus camaldulensis y P. radiata en un brote epidémico ocurrido en Marruecos tras haber agotado el alimento en las masas de quercíneas autóctonas.

En los años 2012 y 2013, en el término municipal de Cubillos del Sil (El Bierzo, León), tuvo lugar un brote epidémico de $L$. dispar que afectó a 46 ha monoespecíficas de $P$. radiata. Este brote presentó la singularidad de haber afectado casi en su totalidad a pino radiata, a pesar de existir en la zona otros huéspedes a priori más adecuados como $Q$. ilex, $Q$. suber y Castanea sativa.

Dado que ese brote se corresponde con uno de los pocos casos documentados de ataque a pino radiata y que la documentación de los brotes anteriores es muy poco detallada, en este trabajo se consideró conveniente analizar los siguientes aspectos:

- Determinar el porqué de la preferencia por el pino radiata como huésped principal en la zona. 
- Estudiar los enemigos naturales de $L$. dispar presentes ante la posibilidad de que la peculiaridad del hábitat le permitiese escapar de su ataque.

- Estimar el efecto de la defoliación en el crecimiento radial.

\section{Material y médotos}

\subsection{Preferencias por la planta huésped}

En la zona de estudio las cuatro especies arbóreas más abundantes son C. sativa, Populus nigra, $Q$. ilex y $P$. radiata, siendo esta última la que ha sido más afectada por el ataque. Para determinar la razón de la elección de $P$. radiata como huésped principal se llevaron a cabo dos estudios paralelos. Por una parte se intentó determinar si este hecho podría explicarse por palatabilidad y calidad nutritiva de las hojas. Para ello, se determinó la dureza de 30 hojas nuevas de cada especie (excepto para $Q$. ilex, donde se consideraron también 30 hojas maduras) con ayuda de un penetrómetro y se analizó el contenido de nitrógeno de 10 hojas por especie por oxidación instantánea de las muestras secadas y molidas. Por otra parte se realizó un bioensayo para determinar la influencia del tipo de alimentación en el desarrollo del insecto. Para ello se tomaron 100 orugas neonatas y se pesaron individualmente; posteriormente se repartieron al azar en cuatro placas Petri y en cada una de las placas se les proporcionaron diariamente hojas de cada una de las cuatro especies arbóreas ensayadas. Al inicio del segundo estado larvario, justo tras la muda, se procedió de nuevo al pesado de las mismas y se anotó la fecha de la muda. El efecto en el desarrollo se cuantificó por tiempo en que tardaron en mudar y el incremento de peso entre ambas medidas.

\subsection{Enemigos naturales}

Para el estudio de los enemigos naturales se procedió a identificar los vertebrados y los insectos depredadores y parasitoides en diferentes fases de desarrollo de L. dispar. Entre los vertebrados sólo se identificaron las aves insectívoras visualizándolas en campo mediante prismáticos. Para identificar las especies de parasitoides de huevos y orugas se recogieron 1360 huevos y 238 orugas. Huevos y orugas se llevaron a laboratorio y se introdujeron en cajas de emergencia que se revisaron periódicamente para identificar y contabilizar los individuos presentes. Los insectos depredadores de huevos y orugas se localizaron directamente en visitas a campo.

\subsection{Efecto de la defoliación en el crecimiento}

Para determinar el efecto de la defoliación en el crecimiento se replantearon y midieron 18 parcelas experimentales en abril de 2014, en la zona afectada por la plaga y en zonas próximas con características similares. Los factores que se tuvie- 
ron en cuenta en el diseño de muestreo fueron el grado de afectación a nivel de masa y la existencia o no de tratamientos selvícolas de clara. Se establecieron tres grados de afectación: la afectación baja o nula se correspondió con parcelas sin defoliación aparente o muy puntual en pies aislados; la afectación media con parcelas que sufrieron una única defoliación, aunque con daños visibles en todos los pies, y la afectación alta con aquellas parcelas que sufrieron defoliaciones dos años consecutivos, y donde la mayoría de los pies estaban total o severamente defoliados. Se instalaron 12 parcelas en masas clareadas (tres con afectación alta, tres con afectación media y seis con afectación baja) y seis en masas no clareadas (tres con afectación alta y otras tres con afectación baja). Cada parcela tenía entre 200 y $500 \mathrm{~m}^{2}$ de superficie e incluyó un mínimo de 25 pies en su interior. Para cada árbol de estas parcelas se midió el diámetro normal y el porcentaje de defoliación. Para ello se establecieron asimismo tres clases: defoliación nula o casi nula: del 0 al 25\%; defoliación media: del 25 al 75\% y defoliación severa: superior al 75\%.Se realizó también una extracción de canutillos de crecimiento (cores) de 15 pies en cada parcela en los que se midió el crecimiento radial. Así se pudo determinar el crecimiento radial previo a la defoliación y durante la defoliación.

En octubre de 2014 se realizó una segunda toma de datos en las 12 parcelas que permanecían en pie (las seis parcelas restantes fueron cortadas para prevenir infestaciones posteriores de perforadores). Las variables medidas fueron el diámetro normal y el nivel de defoliación. Comparando los inventarios realizados en abril y en octubre se determinó el crecimiento en diámetro posterior a la defoliación.

\subsection{Análisis estadístico}

Para establecer las posibles causas de la elección de $P$. radiata como huésped principal se utilizó un análisis de varianza (ANOVA) de un factor, determinando si existían diferencias entre especies para las variables dependientes dureza, contenido en nitrógeno, incremento de peso y duración del primer estado larvario.

En el análisis de la influencia de la defoliación en el crecimiento radial durante el período de defoliación se usó un ANOVA factorial donde los factores fueron las clases de defoliación a nivel de árbol (nula, media, severa) y la presencia o no de tratamientos de claras en la masa. Cuando el efecto de los factores resultó significativo, se incluyeron como covariables el diámetro normal del árbol a la fecha de realización del primer inventario, el crecimiento radial en los últimos 5 años y el índice de competencia BAL relativo (BALr). En ese caso el procedimiento usado fue el análisis de covarianza (ANCOVA).

Para el análisis de la influencia de la defoliación en el crecimiento radial posterior a la defoliación el análisis estadístico fue análogo al anterior, con la diferencia de que las covariables testadas fueron el diámetro normal del árbol a la fecha de realización del segundo inventario y el crecimiento radial durante el período de defoliación.

Cuando el número de niveles de un factor fue superior a dos, se utilizó el test HSD de Tukey de comparaciones múltiples para comparar las medias de los diferen- 
tes niveles del factor. El nivel de significación $(\alpha)$ usado en todo el trabajo fue de 0,05 .

Todos los análisis estadísticos se llevaron a cabo con el programa IBM SPSS Statistics v. 22 (IBM Corp. Released, 2013).

\section{Resultados y Discusión}

\subsection{Preferencia por la planta huésped}

Según los resultados obtenidos en el ANOVA existen diferencias significativas $(p<0,001)$ en la dureza de las hojas y en el contenido en nitrógeno según especies (Fig. 1).

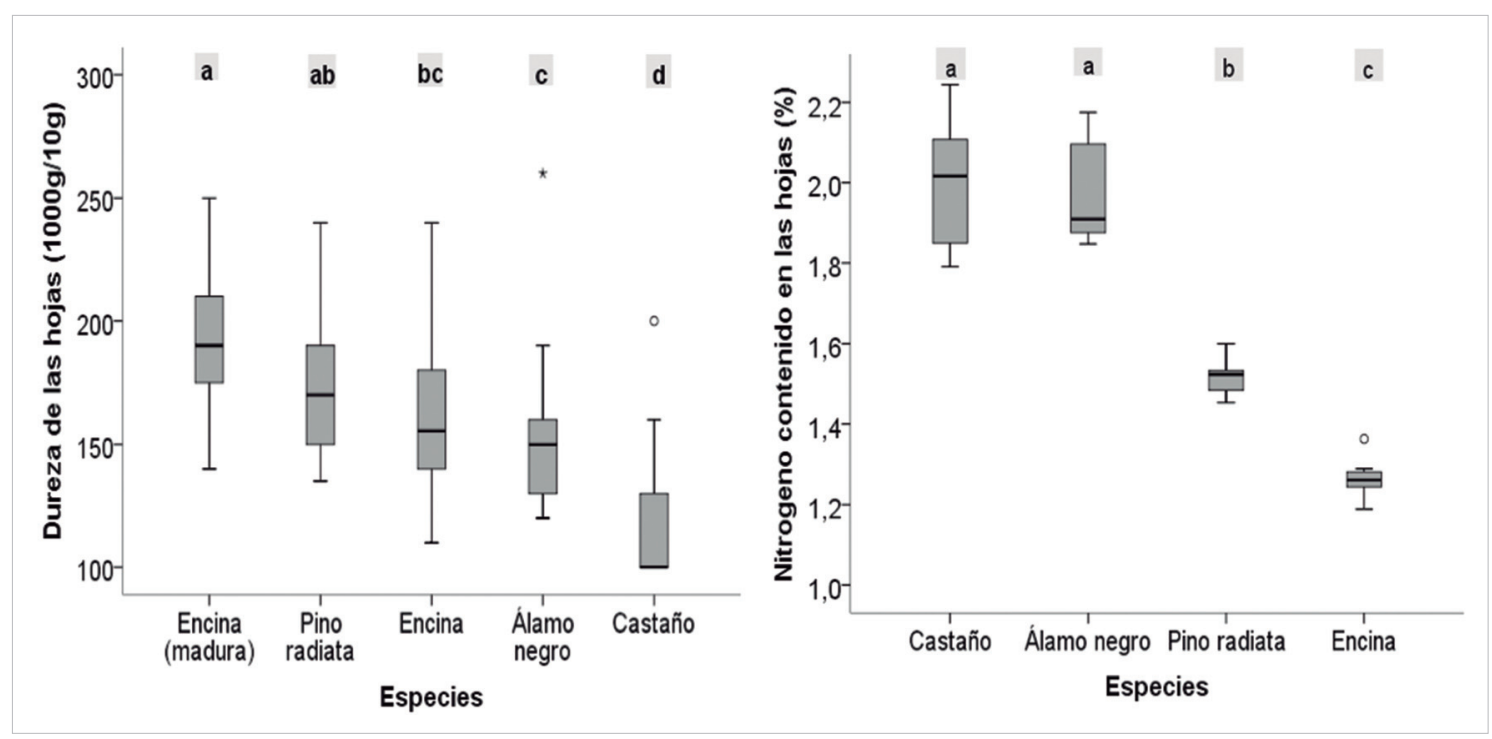

Figura 1. Diagrama de cajas para las variables dureza (izda.) y contenido en nitrógeno (dcha.) de las hojas en función de las especies. Letras diferentes indican diferencias significativas $(p<0,05)$ entre grupos, según el test de Tukey.

La dureza de las hojas es una defensa física de las plantas que afecta al tamaño, supervivencia y procesos adaptativos de los insectos (Larsson and Ohmart, 1988, Johnson and Zalucki, 2007), por lo que es un factor fundamental para el establecimiento de orugas defoliadoras de primer estado en el árbol (Clancy, 2002). Según esta variable, las hojas maduras de encina serían las menos palatables para L. dispar, mientras que el castaño resultaría ser el mejor huésped. Sin embargo, la casi inexistencia de mortalidad durante el primer estado larvario en el bioensayo de alimentación controlada (un 1\% para el total de las 100 orugas) parece indicar que la dureza de las hojas del año de las cuatro especies analizadas no ha sido un factor decisivo en la elección del huésped principal. 
En cuanto a la calidad nutritiva las hojas, el castaño y el álamo negro serían especies, a priori, más susceptibles al ataque de $L$. dispar que el pino radiata y la encina, al poseer un contenido mayor en nitrógeno. Esta variable es clave desde el punto de vista nutricional al ser el nitrógeno un elemento limitado en las plantas (Mattson, 1980; Strong et al., 1984). Joseph et al. (1991), en un ensayo de alimentación controlada con hojas de Pseudotsuga menziesii y Alnus rhombifolia, encontraron que el contenido en nitrógeno explicaba en gran medida la preferencia de $L$. dispar por la frondosa.

Por lo que respecta al bioensayo de alimentación controlada, los resultados del ANOVA indican que existen diferencias significativas en el incremento de peso de las orugas $(p=0,037)$ y en la duración del primer estado larvario $(p<0,001)$ según las especies arbóreas usadas para la alimentación (Fig. 2). El posterior test de Tukey mostró que las orugas que más peso ganaron fueron las que se alimentaron con pino radiata y las que menos (significativamente diferente a las primeras; $p<0,001$ ) fueron las orugas alimentadas con P. nigra (Fig. 2, izda.). Sin embargo, fueron estas orugas, las alimentadas con $P$. nigra, las que completaron el primer estado en menos tiempo, siendo este período significativamente más corto que en las orugas alimentadas con castaño y pino radiata ( $p<0,001$; Fig. 2 , dcha.).
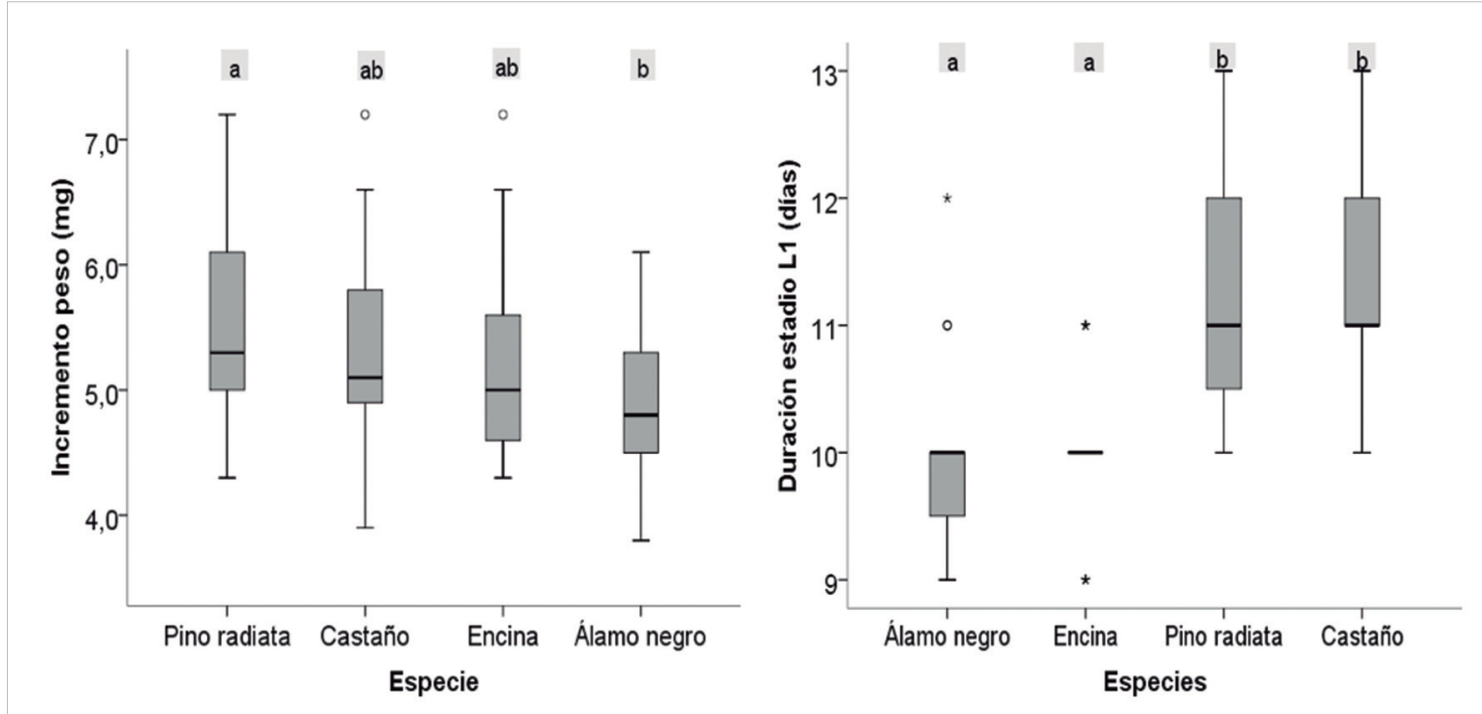

Figura 2. Diagrama de cajas para las variables incremento de peso (izda.) y duración del primer estado larvario (dcha.) en función de las especies. Letras diferentes indican diferencias significativas $(p<0,05)$ entre grupos, según el test de Tukey.

Edwars et al. (1979), en un estudio comparativo con 92 especies arbóreas, obtuvieron mayores incrementos de peso en orugas alimentadas con acículas de coníferas (entre ellas Pseudotsuga menziesii, Picea abies y P. radiata) que con hojas de Q. suber u otras especies del género Quercus. Withers y Keena (2001), por el contrario, muestran un peso 50 veces mayor y un acortamiento de los estados larvarios 
en individuos alimentados con Quercus velutina durante 14 días en comparación con los alimentados con $P$. radiata.

En nuestro estudio, según los análisis foliares, el castaño resultaría ser el huésped más adecuado. Sin embargo, a tenor del mayor incremento de peso en el primer estado larvario, $P$. radiata es, aparentemente, la especie más adecuada para este insecto. Este hecho podría justificarse por la concentración en las acículas de otros compuestos no analizados en el presente estudio como el fósforo, los terpenos, los taninos y los fenoles, que pueden ser determinantes en la selección de huéspedes por parte de L. dispar (Joseph et al., 1991).

Asimismo, el comportamiento observado en la zona de estudio podría estar influenciado por otras características de palatabilidad o el contenido hídrico de las hojas. Estos factores están relacionados con la fenología de brotación y desarrollo de las hojas, y que puede tener un gran efecto sobre la supervivencia y crecimiento de las orugas (Raupp et al., 1988; Valentine, 1983). Según las observaciones realizadas en campo, la encina y el castaño son las dos especies menos coordinadas fenológicamente con la eclosión de los huevos de L. dispar, debido a su brotación más tardía, lo que obligaría a las orugas recién nacidas a desplazarse por un mayor número de brotes para alimentarse de las hojas tiernas.

\subsection{Enemigos naturales}

Se constató la presencia de numerosos enemigos naturales de L. dispar que contribuyeron en mayor o menor medida al declive de la población en el año 2014. A pesar de lo peculiar del hábitat estudiado, las especies localizadas fueron similares a las citadas en masas de frondosas en la Península Ibérica e Islas Baleares (Govern de les Illes Balears, 2010; Ceia y Ramos, 2014), excepto por la especie Dermestes erichsoni. Este resultado sugiere que $P$. radiata no ha interferido en la localización de $L$. dispar por parte de enemigos naturales, aunque sí parece influir en el nivel de parasitismo de algunas especies.

Las especies de enemigos naturales identificadas en este estudio aparecen en la Tab. 1 .

Tabla 1. Listado de especies de enemigos naturales de $L$. dispar identificadas en este estudio.

\begin{tabular}{l|l|l|l}
\hline Orden & Familia & Especie & Hábito \\
\hline \multirow{4}{*}{ Hymenoptera } & $\begin{array}{l}\text { Encyrtidae } \\
\text { Eupelmidae }\end{array}$ & $\begin{array}{l}\text { Ooencyrtus kuvanae Howard } \\
\text { Anastatus japonicus Ashmead }\end{array}$ & $\begin{array}{l}\text { Parasitoide de huevos } \\
\text { Parasitoide de huevos }\end{array}$ \\
\cline { 2 - 4 } & $\begin{array}{l}\text { Braconidae } \\
\text { Tachinidae }\end{array}$ & $\begin{array}{l}\text { Cotesia melanoscela } \text { (Ratz.) } \\
\text { Compsilura concinnata } \text { Meigen }\end{array}$ & $\begin{array}{l}\text { Parasitoide de larvas } \\
\text { Parasitoide de larvas }\end{array}$ \\
\hline \multirow{2}{*}{ Coleoptera } & Carabidae & Calosoma sycophanta L. & Depredador \\
\cline { 2 - 4 } & Dermestidae & Dermestes erichsoni Ganglbauer & Desmantelador de puestas \\
\hline
\end{tabular}


Se identificaron además 13 especies de aves insectívoras y se observaron síntomas de la enfermedad causada por el virus denominado Nuclear Polyhedrosis Virus (NPV) en larvas de L. dispar. Asimismo se detectó Dibrachys cavus Walker, un hiperparasitoide de Cotesia melanoscela.

El impacto en las poblaciones de L. dispar de las especies parásitas de huevos fue variable. En el caso de O. kuvanae y A. japonicus el porcentaje de parasitismo osciló entre un $3,7 \%$ y un $18,2 \%$ entre puestas, con un promedio de un $11,6 \%$. Esta variabilidad observada parece ser una constante en los distintos estudios publicados. Así Brow (1984) y Alalouni et al. (2013), en revisiones a nivel mundial, comprobaron que el porcentaje de parasitismo de $O$. kuvanae oscila entre 10 y $40 \%$, mientras que Riba y Pujalte (2008) en condiciones mediterráneas muestran valores entre el 30 y el 50\%. En el caso de A. japonicus, Alalouni et al. (2013) indican porcentajes medios de parasitismo entre 10 y $20 \%$ en Europa.

El porcentaje medio de parasitismo en los estados larvarios de $L$. dispar por $C$. melanoscela observados en el presente estudio fue de un $4,2 \%$, datos que coinciden con lo observado por otros autores (Fuester et al., 1983; Falcó et al., 1990). El porcentaje de parasitismo por parte de representantes de la familia Tachinidae fue del $6,7 \%$.

\subsection{Efecto de la defoliación en el crecimiento}

Según los resultados del ANOVA factorial, existen diferencias significativas en el crecimiento radial durante el período de defoliación (años 2012 y 2013) según el nivel de defoliación $(p<0,001)$. La inclusión en el ANOVA de las covariables citadas en el Apartado 2.4. no resultó significativa ( $p>0.05$ para todas las covariables), como tampoco lo fue el efecto de los tratamientos de clara $(p=0,155)$ ni la interacción entre los efectos de clara y defoliación $(p=0,407)$. Estos resultados parecen, a priori, coherentes. Así, era esperable que los árboles con defoliación severa sufrieran una reducción en el crecimiento mayor que los árboles con defoliación menos intensa debido a la diferente reducción de área fotosintética sufrida (Jacquet et al., 2012). En cuanto a la clara, probablemente no afectó al crecimiento radial porque se llevó a cabo el año del comienzo del brote epidémico (febrero de 2012) y los árboles remanentes no pudieron aprovechar el espacio proporcionado por el tratamiento selvícola para incrementar su vigor.

Estos resultados quedan refrendados visualmente en los diagramas de cajas: los árboles con los niveles de defoliación nula y media (0-25\% y 25-75\%) crecieron más que los árboles con defoliación severa (75-100\%) (Fig. 3, izda.); los árboles situados en parcelas clareadas crecieron prácticamente igual que los situados en parcelas sin clarear (Fig. 3, dcha.).

En cuanto al crecimiento radial post-defoliación, los resultados del ANOVA muestran que no existen diferencias significativas según el factor clara $(p=0,549)$. Sin embargo al realizar un ANCOVA considerando este mismo factor y añadiendo el diámetro normal como covariable, ésta fue significativa $(p<0,001)$ y afectó al nivel de significación del factor, que pasó a ser significativo $(p=0,026)$. Los resul- 


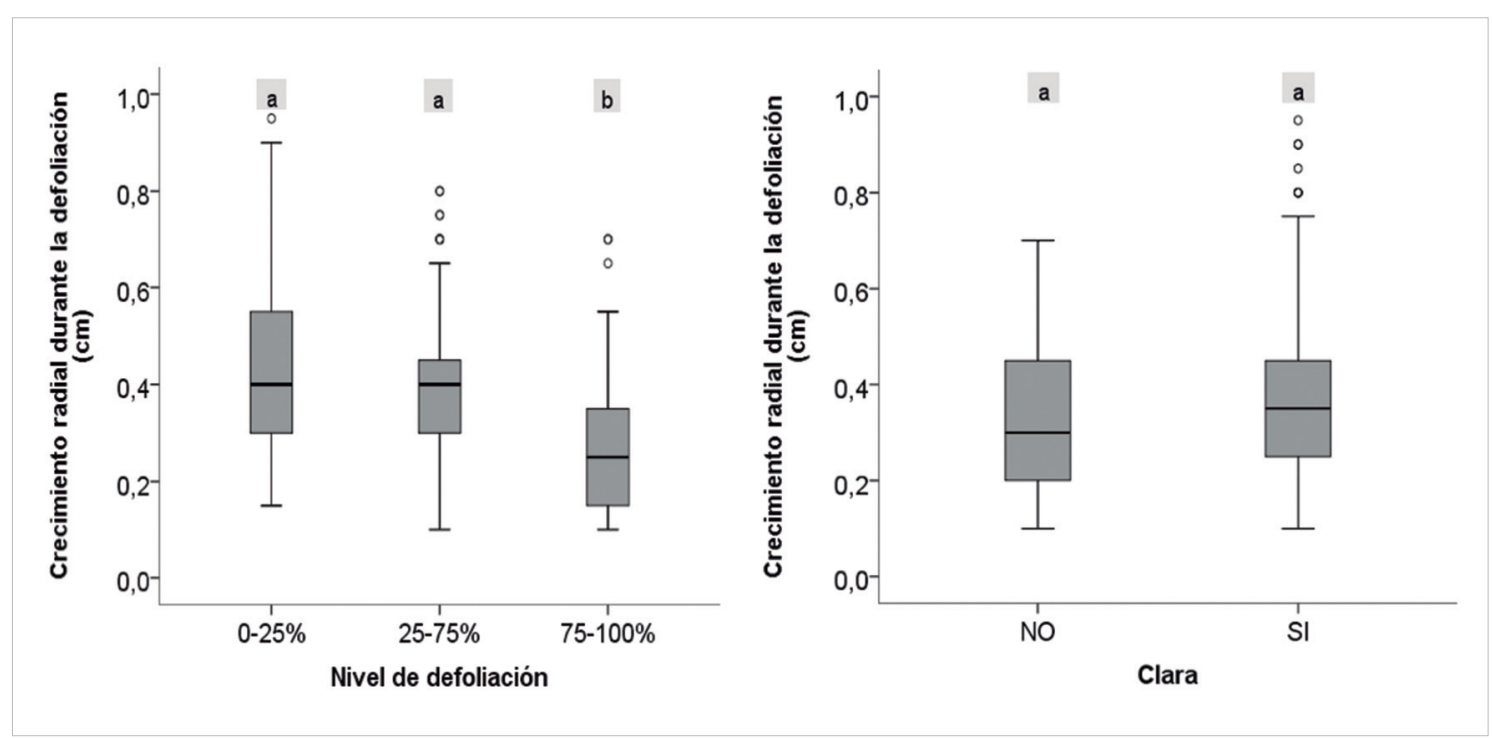

Figura 3. Diagramas de cajas para la variable crecimiento radial durante el periodo de defoliación en función del nivel de defoliación (izda.) y de la realización de tratamiento selvícola de clara (dcha.). Letras diferentes indican diferencias significativas $(p<0,05)$ entre grupos, según el test de Tukey.

tados parecen indicar que la clara sí fue determinante en el crecimiento radial postdefoliación. Este hecho se explica porque los árboles situados en zonas clareadas tienen una mayor capacidad para el incremento de la biomasa foliar al existir una menor competencia intraespecífica.

Por otro lado se verificó que existen diferencias significativas en el crecimiento radial post-defoliación según el factor nivel de defoliación $(p<0,05)$. Al realizar un ANCOVA considerando el diámetro normal como covariable, se comprobó que

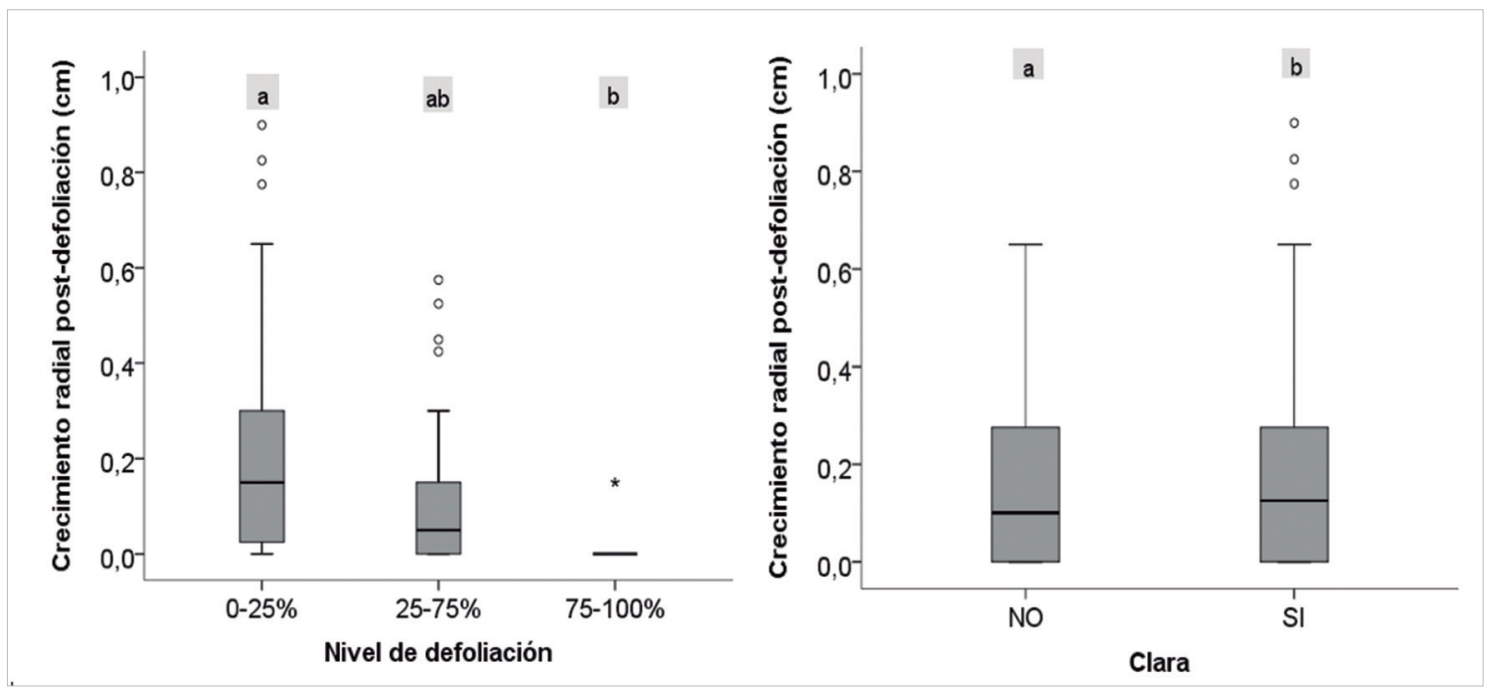

Figura 4. Diagramas de cajas para la variable crecimiento radial post-defoliación en función del nivel de defoliación (izda.) y de la existencia o no de clara (dcha.). Letras diferentes indican diferencias significativas $(p<0,05)$ entre grupos, según el test de Tukey. 
ésta no afecta a la significación del nivel de defoliación. Era esperable que los árboles con defoliación severa presentaran un crecimiento radial menor tras la defoliación, ya que los árboles más afectados tienen que invertir más tiempo y recursos en la recuperar la copa. El test de Tukey para el factor nivel de defoliación puso de manifiesto que existen diferencias significativas entre el nivel de defoliación severo $(75-100 \%)$ y el nivel de defoliación nulo $(0-25 \%)(p<0,05)$. Este resultado confirma resultados previos relativos a $L$. dispar, en los que se verificó que la pérdida de crecimiento persiste el año siguiente de la defoliación (Baker 1941; Kucherov 1991; Huber 1993; Muzika y Liebhold 1999), dilatándose incluso durante más de tres años (Fratzian 1973; Magnoler y Cambini 1973; Twery 1987).

Los resultados del ANOVA quedan refrendados visualmente mediante diagramas de cajas: los árboles con un nivel de defoliación más bajo tienen un crecimiento radial post-defoliación mayor que los árboles con defoliación más intensa (Fig. 4, $i z d a$.); los árboles en zonas clareadas mostraron un crecimiento radial mayor que los árboles en zonas sin clarear (Fig. 4, dcha.).

\section{Conclusiones}

En la zona de estudio se observó una clara preferencia de L. dispar por masas de $P$ radiata, así como un mayor incremento de peso durante el primer estado larvario en larvas alimentadas artificialmente con hojas de esta especie. Sin embargo, los resultados del análisis de variables relacionadas con el valor nutricional y de palatabilidad de las hojas, como el contenido de nitrógeno o la dureza, no parecen justificar estos comportamientos. Compuestos químicos foliares no analizados o la fenología de la brotación y desarrollo de las hojas de las especies alternativas en la zona podrían explicar la preferencia y mayor desarrollo sobre $P$. radiata.

A pesar de lo peculiar del hábitat estudiado, las especies de enemigos naturales de $L$. dispar localizadas fueron similares a las citadas para frondosas en otras zonas de la Península Ibérica.

El crecimiento radial de $P$. radiata se vio afectado por el nivel de defoliación; los árboles con un mayor nivel de defoliación crecieron significativamente menos que aquellos menos defoliados, tanto durante el período de defoliación como tras el mismo. Las claras no han jugado un papel destacable en el crecimiento durante el período de defoliación, pero sí posteriormente, de lo que se infiere que este tratamiento selvícola puede facilitar la capacidad de recuperación de la biomasa foliar tras la defoliación.

\section{Agradecimientos}

Este trabajo fue financiado por el contrato de investigación "Seguimiento y bases para la gestión de las masas forestales afectadas por defoliación de Lymantria dispar en Fresnedo (Cubillos del Sil) empleando SIG y Teledetección", financiado por el Ayuntamiento de Cubillos del Sil. 
La identificación de los himenópteros fue refrendada por el Dr. Juli Pujade Villar, de la Universidad de Barcelona.

Asimismo, agradecemos la colaboración de los Agentes Medioambientales de la zona, en especial de Agustín Luis Blanco.

\section{Bibliografía}

Alalouni, U., Schädler, M., Brandl, R., 2013. Natural enemies and environmental factors affecting the population dynamics of the gypsy moth. J. Appl. Entomol. 137, 721-738.

Avci, M., 2009. Parasitoid complex and new host plants of the gypsy moth, Lymantria dispar L. in the lakes district, Turkey. J. Anim. Vet. Adv. 8, 1402-1405.

Baker, W.L., 1941. Effect of gypsy moth defoliation on certain trees. J. For. 39, 1017-1022.

Brown, J.H., Cruikshank, V.B., Gould, W.P., Husband, T.P., 1988. Impact of gypsy moth defoliation in stands containing white pine. North. J. Appl. For. 5, 108-111.

Brown, M.W., 1984. Literature review of Ooencyrtus kuvanae (Hym: Encyrtidae), an egg parasite of Lymantria dispar (Lep: Lymantriidae). Entomophaga 29, 249-265.

Ceia, R.S., Ramos, J.A., 2014. Birds as predators of cork and holm oak pests. Agrofor. Syst. pp 1-18. DOI 10.1007/s10457-014-9749-7.

Clancy, K.M., 2002. Mechanisms of resistance in trees to defoliators. In: Wagner, M.R., Clancy, K.M., Lieutier, F., Paine, T.D. (eds.), Mechanisms and deployment of resistance in trees to insects. Kluwer Academic Publishers, The Netherlands, pp. 77-101.

Closa, A., Nuñez, L., 2009. La plaga de la lagarta peluda Lymantria dispar L. (Lep.: Lymantridae) en la isla de Menorca (2003-2008). En: S.E.C.F.-Junta de Castilla y León (Eds.). Actas $5^{\circ}$ Congreso Forestal Español. CD-Rom. Sociedad Española de Ciencias Forestales. Ávila.

Edwars, J.G., Fusco, R.A., 1979. Gypsy moth larva host plant screening and evaluation. A Final Research Report to California Department of Food and Agriculture, pp. 16.

Eisenbies, H.M., Davidson, C., Johnson, J., Amateis, R., Gottschalk, K., 2007. Tree mortality in mixed pine-hardwood stands defoliated by the European gypsy moth (Lymantria dispar L.). For. Sci. 53, 683-691.

Falcó, J.V., Luna, F., Jiménez, R. 1990. Complejo parasitario de Lymantria dispar L. en Sierra Espadán Castellón (Lepidoptera: Lymantriidae). Bol. Asoc. Esp. Ent. 14, 261-267.

Fratzian, A., 1973. Growth and vitality of oak stands after being eaten by gypsy moths, $L y-$ mantria dispar L., in Romania. Anzeiger für Schädlingskunde, Pflanzenschutz, Umweltschutz 46, 122-125.

Fraval, A., 1989. Degats et nuisances. In: Fraval, A. (ed.), Lymantria dispar pp. 73-76. http: //www7.inra.fr/dpenv/ld-m-deg.htm. Accessed 25 April 2015.

Fuester, R.W., Drea Jr, J.J., Gruber, F., Hoyer, H., Mercadier, G., 1983. Larval parasites and other natural enemies of Lymantria dispar (Lepidoptera: Lymantriidae) in Burgenland, Austria, and Würzburg, Germany. Environ. Entomol. 12, 724-737.

Govern de les Illes Balears., 2010. Vectores biológicos de Lymantria dispar en los encinares Menorquines.http://www.caib.es/sacmicrofront/noticia.do?mkey=M149\&cont=67136\& lang=ES Accessed 20 March 2015.

Huber, F., 1993. Determinisme de la surface des vaisseaux du bois des chenes indigenes 
(Quercus robur L., Quercus petraea Liebl.): effet individuel, effet de 1'appareil foliaire, des conditions climatiques et de l'age de l'arbre. Ann. For. Sci. 50, 509-524.

IBM Corp. Released., 2013. IBM SPSS Statistics for Windows, Version 22.0. Armonk, NY: IBM Corp.

Jacquet, J.S., Orazio, C., Jactel, H., 2012. Defoliation by processionary moth significantly reduces tree growth: a quantitative review. Ann. For. Sci. 69, 857-856.

Johnson M.L., Zalucki M.P., 2007. Feeding and foraging behaviour of a generalist caterpillar: are third instars just bigger versions of firsts? Bull. Entomol. Res. 97, 81-8.

Joseph, G., Miller, J.C., Berry, R.E., Wernz, J., Moldenke, A.F., Kelsey, R.G., 1991. White alder and Douglas-fir foliage quality and interegg-mass influences on larval development of gypsy moth, Lymantria dispar. J. Chem. Ecol. 17, 1783-1799.

Kucherov, S., 1991. The reconstruction of Lymantria dispar outbreaks by dendrochronological methods in the south Urals. In: Baranchikov, Y.N., Mattson, W.J., Hain F.P., Payne, T.L. (eds.), Forest insect guilds: patterns of interaction with host trees. USDA Forest Service Gen. Tech Rep. NE-153. pp. 205-206.

Larsson S., Ohmart C.P., 1988. Leaf age and larval performance of the leaf beetle, Paropsis atomaria. Ecol. Entomol. 13, 19-24.

Leites, R.M.M.R., 1993. Ocorrência de Lymantria dispar L. em Pinus radiata D. Don: estudo do ciclo de vida e comportamento da praga neste hospedeiro: medidas de protecçäo e combate. Tesis de Maestría. Instituto Politécnico de Castelo Branco.

Liebhold, A.M., Gottschalk, K.W., Muzika, R.M., Montgomery, M.E., Young, R., O`Day, K., Kelley, B., 1995. Suitability of North American tree species to the gypsy moth: a summary of field and laboratory test. USDA Forest Service Gen. Tech Rep. NE-211, 34 pp.

Magnoler, A., Cambini, A., 1973. Radial growth of cork oak and the effects of defoliation caused by larvae of Lymantria dispar L. and Malacosoma neustria L. Boletim do Instituto dos Produtos Florestais, Cortica 35, 53-59.

Mattson, W.J., 1980. Herbivory in relation to plant nitrogen content. Ann. Rev. Ecol. Syst. 11, 119-161.

Muñoz, C., Pérez, V., Cobos, P., Hernández, R., Sánchez, G., 2011. Sanidad forestal: guía en imágenes de plagas enfermedades y otros agentes presentes en los bosques. Ediciones Mundi-Prensa, $3^{\mathrm{a}}$ edición. Madrid.

Muzika, R.M., Liebhold, A.M., 1999. Changes in radial increment of host and nonhost tree species with gypsy moth defoliation. Can. J. For. Res. 29, 1365-1373.

Raupp, M.J., Werren, J.H., Sadof, C.S., 1988. Effects of short-term phonological changes in leaf suitability on the survivorship, growth and development of gypsy moth (Lepidoptera: Lymantriidae) larvae. Environ. Entomol.17, 316-19.

Riba i Flinch, J.M., Pujade i Villar, J., 2008. Colecta de puestas de Lymantria dispar L. (Lep.: Lymantriidae) en zonas tratadas y no tratadas químicamente en los encinares de Menorca y estudios de los parasitoides de huevos: perspectivas de futuro. Universidad de Barcelona. http://www.caib.es/sacmicrofront/archivopub.do?ctrl=NTCS025187ZI62898 \&id=62898. Accessed 20 March 2015.

Romanyk, N., 1973. Les gradations de Lymantria dispar L. en Espagne. Zastita Bilja 124$125,285-288$.

Romanyk, N., Rupérez, A., 1960. Principales parásitos observados en los defolidores de España con atención particular de la Lymantria dispar L. Entomophaga 5, 229-236.

Strong, D.R., Lawton, J.H., Southwood, T.R.E., 1984. Insects on plants: community patterns 
and mechanisms. Harvard University Press, $313 \mathrm{pp.}$

Tobin, P.C., Liebhold, A.M., 2011. Gypsy moth. In: Simberloff, D., Rejmanek, M. (eds.), Encyclopedia of Biological Invasions, University of California Press, pp. 298-304.

Twery, M.J., 1987. Changes in the vertical distribution of xylem production in hardwoods defoliated by gypsy moth. Tesis Doctoral. Yale University.

Twery, M.J., 1990. Effects of defoliation by gypsy moth. In: Gottschalk, K.W., Twery, M.J., Smith, S.I. (eds.), Gypsy moth research review 1990. USDA Forest Service Gen. Tech Rep. NE-146. 27-39 pp.

Valentine, H.T., 1983. Budbreak and leaf growth functions for modeling herbivory in some gypsy moth hosts. For. Sci. 29, 607-17.

Withers, T.M., Keena, M.A., 2001. Lymantria monacha (nun moth) and L. dispar (gypsy moth) survival and development on improved Pinus radiata. N. Z. J. For. Sci. 31, 66-77. 
